\title{
Medical treatment of endometriosis: an update
}

\author{
Laila Ezzat* \\ Department of Obstetrics and Gynecology, Aswan University, Aswan, Egypt
}

Received: 20 June 2017

Accepted: 19 July 2017

\section{*Correspondence:}

Dr. Laila Ezzat,

E-mail: lailaezzat972000@gmail.com

Copyright: $\odot$ the author(s), publisher and licensee Medip Academy. This is an open-access article distributed under the terms of the Creative Commons Attribution Non-Commercial License, which permits unrestricted non-commercial use, distribution, and reproduction in any medium, provided the original work is properly cited.

\begin{abstract}
Endometriosis is defined as the presence of endometrial-like tissue (glands and stroma) outside the uterus, which induces a chronic inflammatory reaction, scar tissue, and adhesions that may distort a woman's pelvic anatomy. Endometriosis is primarily found in young women, but its occurrence is not related to ethnic or social group distinctions. Patients with endometriosis mainly complain of pelvic pain, dysmenorrhea, and dyspareunia. Endometriosis is a very common debilitating disease that occurs in 6 to $10 \%$ of the general female population; in women with pain, infertility, or both, the frequency is $35-50 \%$. This is an evidence based narrative review conducted by searching Medline up to (2017) and other online articles from Pubmed, Google scholar by using terms like Endometriosis, management, evidence based, updated treatment, pharmacoceutical hormone treatment and nonhormonal treatment. Articles were selected based on their currency and relevance to the discussion. Numerous cofactors can play a role in the progression and maintenance of endometriosis, which is thus a complex multifactorial disease. Hormonal therapy represents the treatment of choice in patients with endometriosis; with this in mind, we could consider CAM a supplementary option to be added to hormonal treatment, or as a valuable opportunity for those women in whom medical therapy is contraindicated.
\end{abstract}

Keywords: Endometriosis, Evidence based, Management, Pharmacoceutical hormone treatment, Non-hormonal treatment, Updated treatment

\section{INTRODUCTION}

Endometriosis is defined as the presence of endometriallike tissue (glands and stroma) outside the uterus, which induces a chronic inflammatory reaction, scar tissue, and adhesions that may distort a woman's pelvic anatomy. ${ }^{1}$

Endometriosis is primarily found in young women, but its occurrence is not related to ethnic or social group distinctions. Patients with endometriosis mainly complain of pelvic pain, dysmenorrhea, and dyspareunia. ${ }^{2}$ The associated symptoms can impact the patient's general physical, mental, and social well-being. ${ }^{1}$ Endometriosis is a very common debilitating disease that occurs in 6 to $10 \%$ of the general female population; in women with pain, infertility, or both, the frequency is $35-50 \% .^{3}$ Clinical presentations of endometriosis often consist of chronic pelvic pain and infertility; however, many patients are asymptomatic. ${ }^{3}$

The earliest visible manifestations of endometriosis are whitish peritoneal plaques. The foci of endometrial tissue are small subserosal nodules with a brown appearance on gross examination; termed powder burns, they are seen on laparoscopic examination.

Over time, the repeated hemorrhaging can produce extensive fibrosis surrounding the endometrial tissue, which can result in adhesions to adnexal structures or to bowel and can obliterate the pelvic cul-de-sac. ${ }^{4}$ 
When the ovaries are involved, they can become enlarged with cystic, blood-filled spaces that, on gross examination, are termed chocolate cysts, or endometriomas. Endometriomas can become large and multilocular. $^{4}$

To date, it has not been possible to determine whether a medical approach is less expensive than a surgical approach in patients with chronic pelvic pain. Also, data is lacking regarding the cost of treating endometriosis in infertile patients. ${ }^{5}$

This review focuses on the most recent discoveries regarding medical treatment of endometriosis. This is an evidence based narrative review conducted by searching Medline up to (2017) and other online articles from Pubmed, Google scholar by using terms like Endometriosis, management, evidence based, updated treatment, pharmacoceutical hormon treatment and nonhormonal treatment. Articles were selected based on their currency and relevance to the discussion. We did not use statistical testing or concepts of statistical significance

Current article would provide an approach to best practice management of endomrtriosis and also promote and facilitate a more educated, systematic and effective physician response. Reviewed articles were restricted to English language only. Studies in both animals and humans were reviewed for the manuscript.

\section{The management options of endometriosis}

Endometriosis should be viewed as a chronic disease characterized by pelvic pain and associated with infertility. It requires a life-long personalized management plan with the goal of maximizing medical treatment and avoiding repeated surgical procedures. The treatment for endometriosis is essentially chosen by each individual woman, depending on symptoms, age, and fertility. For many women, adequate treatment requires a combination of treatments given over their lifetime. The current treatments include medical, surgical, or a combination of these approaches.

A combination of surgical treatment and either preoperative or postoperative medical therapy has been suggested for endometriosis. Surgical treatment followed by medical treatment may prolong the pain-free (or reduced-pain) interval compared to surgery alone. However, there is insufficient evidence to support the conclusion that hormonal suppression in association with surgery for endometriosis is associated with a significant benefit in pain recurrence or infertility.

Endometriosis-associated pain has been well studied, and all the established medical therapies provided a better outcome than placebo. ${ }^{6}$ However, none seems to be markedly better than another. studies have demonstrated significant differences in the efficacies of medical and surgical therapies. ${ }^{7,8}$

\section{Conventional medical treatment for endometriosis}

Oral contraceptives (OC), progestogens, danazol, GnRHa, and anti-progestogens all have been used for the treatment of endometriosis. ${ }^{9}$ Clinical trials involving such treatments are difficult because they routinely result in amenorrhea, and some result in hypoestrogenic effects that interfere with efforts to perform a blinded study. No studies have compared directly medical versus surgical treatment of endometriosis, and thus there is no substantial evidence to establish the superiority of one approach than the other. Costs and side effects often dictate the choice of medical treatment. ${ }^{10}$

\section{Combined hormonal contraceptives}

Combined hormonal contraceptives have been used in both a cyclic and a continuous fashion in the treatment of symptoms associated with endometriosis. Decidualization followed by atrophy of the endometrial tissue is the proposed mechanism of action. ${ }^{11}$ Whereas combined OCs containing the more androgenic progestogens (19nortestosterone derivatives) traditionally have been used to treat endometriosis symptoms, combined OCs containing the new generation progestogen, desogestrel, also have proven effective. ${ }^{12}$ A low-dose combined OC administered in a cyclic regimen to women with endometriosis was found as effective as GnRH-a treatment for relief of dyspareunia and non-menstrual pain as assessed by a pain scoring system. ${ }^{13}$ However, GnRH-a treatment was more effective than combined OCs for the relief of dysmenorrhea because the agonist reliably induces amenorrhea. ${ }^{13}$ A prospective observational trial demonstrated that continuous low-dose combined OCs were more effective than cyclic combined OCs in controlling endometriosis symptoms in patients after surgical treatment for endometriosis. ${ }^{14}$

\section{Progestogens}

In observational studies involving treatment with medroxyprogesterone acetate (MPA), dydrogesterone, or norethindrone acetate, pain has been reduced by $70 \%$ $100 \% .^{15}$ A meta-analysis of four randomized, controlled trials comparing MPA to danazol alone, danazol and combined OCs, or a GnRH-a.

The levonorgestrel-releasing intrauterine system (LNGIUS) represents another approach to the medical goserelin acetate) concluded that MPA was as effective as the other treatments (odds ratio OR 1.1; 95\% CI 0.43.1). ${ }^{15}$ Randomized studies concluded that dienogest was significantly better than placebo and as effective as the GnRH-a buserelin, LA, or triptorelin in reducing pain symptoms with diminished side effects of hot flushes and bone mineral density loss treatment of endometriosis. ${ }^{16} \mathrm{~A}$ randomized, controlled trial comparing the LNG-IUS to expectant management after laparoscopic surgical treatment for symptomatic endometriosis found that the LNG-IUS was more effective than no treatment in 
reducing symptoms of dysmenorrhea. ${ }^{17}$ Other studies have demonstrated improved symptoms associated with rectovaginal endometriosis and a significant decrease in the extent of disease observed at second-look laparoscopy after 6 months of treatment with the LNG-IUS. ${ }^{18,19}$ Relief of endometriosis pain with the LNG-IUS is similar to GnRH-a. ${ }^{20}$

\section{GnRH agonists}

Gonadotropin-releasing hormone agonist treatment for endometriosis has been studied more extensively than other medical treatment regimens. Gonadotropinreleasing hormone agonists are modified forms of $\mathrm{GnRH}$ that bind to receptors in the pituitary but have a longer half-life than native GnRH and thereby result in downregulation of the pituitary-ovarian axis and hypoestrogenism. The likely mechanism of action for relief of endometriosis pain involves the induction of amenorrhea and progressive endometrial atrophy. ${ }^{10}$

A Cochrane analysis found that GnRH-a were more effective than placebo for endometriosis pain relief but were similar to the LNG-IUS and danazol. ${ }^{21}$ A long-term follow-up study of patients treated with a GnRH-a alone for 6 months revealed a 53\% recurrence of disease/symptoms 2 years after treatment. ${ }^{22}$

To reduce negative effects of $\mathrm{E}$ deprivation (e.g., bone loss, hot flushes) and allow for longer treatment periods, "add-back" therapy with norethindrone acetate or a combination of $\mathrm{E}$ and progestogen has been advocated. This treatment regimen decreases bone loss seen with GnRH-a alone and also reduces the severity of hypoestrogenic side effects associated with GnRH-a treatment. The underlying theory of add-back treatment is the "E threshold hypothesis,", which holds that the amount of E and/or progestogen necessary to prevent hot flushes, bone loss, and other hypoestrogenic symptoms and side effects is less than that which would stimulate endometriosis. $^{23}$ Although norethindrone acetate is the only hormone approved by the US Food and Drug Administration for add-back therapy, other combinations of low-dose $\mathrm{E}$ and progestogens also have been shown to be effective in decreasing hypoestrogenic side effects and maintaining bone density, and not adversely affecting the extent of pain relief achieved with GnRH-a treatment. ${ }^{21}$ The add-back therapy should be started at the same time as the agonist rather than delaying until a period of hypoestrogenism has occurred. This approach has been shown to decrease bone loss and improve vasomotor symptoms and compliance. ${ }^{24}$

\section{Danazol}

Danazol is a derivative of $17 \alpha$-ethinyltestosterone and acts primarily by inhibiting the LH surge and steroidogenesis and by increasing free $\mathrm{T}$ levels. ${ }^{11}$ Hyperandrogenic side effects are common and include hirsutism, acne, weight gain, and deepening of the voice. ${ }^{25}$ Typically this medication is administered orally; however, vaginal administration as well as vaginal and intrauterine delivery systems have been reported. ${ }^{26-29}$ When compared with placebo, danazol treatment was effective in relieving painful symptoms due to endometriosis, and laparoscopic scores improved. Danazol provided comparable pain relief to GnRH-a but was not as well tolerated. ${ }^{21}$

\section{Gestrinone}

Gestrinone (ethylnorgestrienone, R2323) is an antiprogestational steroid used in Europe for the treatment of endometriosis, but it is not currently available in the United States. ${ }^{30}$ The mechanism of action includes a progestational withdrawal effect at the endometrial cellular level and inhibition of ovarian steroidogenesis. ${ }^{11}$ The drug is administered orally daily to weekly with doses ranging from $2.5-10 \mathrm{mg}$. Side effects relate to both androgenic and antiestrogenic effects. Gestrinone was shown to be as effective as danazol and GnRH analogues. ${ }^{31}$

\section{Aromatase inhibitors}

In several studies involving small numbers of patients, aromatase inhibitors have been shown to be effective for the treatment of endometriosis and pelvic pain in premenopausal and postmenopausal women. ${ }^{24,32}$

A randomized trial of women on goserelin treated with anastrozole or placebo reported no difference in symptom scores during treatment, but the anastrozole group had a lower recurrence rate as well as a longer time to symptom recurrence. ${ }^{33}$ However, anastrozole increased bone loss compared with goserelin alone. ${ }^{33}$ In premenopausal women aromatase inhibitors lead to an increase in FSH levels and subsequent follicular development and therefore must be used in combination with additional agents (progestogens, combined OCs, or GnRH-a) to down-regulate the ovaries. The combination of an aromatase inhibitor with a combined OC may improve endometriosis pain while suppressing follicle development and preserving bone mineral density. ${ }^{24}$

\section{Other medication}

Opioids: Morphine sulphate tablets and other opioid painkillers work by mimicking the action of naturally occurring pain-reducing chemicals called "endorphins". There are different long acting and short acting medications that can be used alone or in combination to provide appropriate pain control.

Following laparoscopic surgery women who were given Chinese herbs were reported to have comparable benefits to women with conventional drug treatments, though the journal article that reviewed this study also noted that "the two trials included in this review are of poor methodological quality so these findings must be 
interpreted cautiously. Better quality randomised controlled trials are needed to investigate a possible role for CHM (Chinese Herbal Medicine) in the treatment of endometriosis. ${ }^{34}$

Pentoxifylline, an immunomodulating agent, has been theorized to improve pain as well as improve pregnancy rates in women with endometriosis. A 2012 Cochrane review, however, found that there was not enough evidence to support the effectiveness or safety of either of these uses. ${ }^{35}$ Current American Congress of Obstetricians and Gynecologists (ACOG) guidelines do not include immune-modulators, such as pentoxifylline, in standard treatment protocols. ${ }^{36}$

Angiogenesis inhibitors lack clinical evidence of efficacy in endometriosis therapy under experimental in vitro and in vivo conditions, compounds that have been shown to exert inhibitory effects on endometriotic lesions include growth factor inhibitors, endogenous angiogenesis inhibitors, fumagillin analogues, statins, cyclooxygenase-2 inhibitors, phytochemical compounds, immunomodulators, dopamine agonists, peroxisome proliferator-activated receptor agonists, progestins, danazol and gonadotropin-releasing hormone agonists. ${ }^{37}$ However, many of these agents are associated with undesirable side effects and more research is necessary. An ideal therapy would diminish inflammation and underlying symptoms without being contraceptive. ${ }^{38,39}$

The overall effectiveness of manual physical therapy to treat endometriosis has not yet been identified. ${ }^{40}$ There is no evidence to support nutritional therapy as effective.

Although hormonal therapies represent the cornerstone in the management of endometriosis, the possible adjuvant role of other remedies and behaviors, such as diet, dietary supplements, physical exercise, osteopathy, acupuncture, and Chinese herbal medicine (CHM), could represent a complementary and feasible approach in the treatment of symptoms related to the disease. ${ }^{41,42}$ Complementary and alternative medicine (CAM) has been used extensively in most Asian countries since the nineteenth century; however, their utilization in Western countries has been rapidly growing. ${ }^{43,44}$

As stated by the World Health Organization, CAM comprises a range of different health-related strategies that can be defined as predominantly operating outside the conventional medical curriculum and profession. ${ }^{45}$ The list of CAMs includes several procedures and products that have been tested in the treatment of women with endometriosis, such as osteopathic manipulative therapy (OMT), massages, acupuncture, transcutaneous electrical nerve stimulation (TENS), CHM, vitamins, and dietary supplementations.

Symptomatic women with endometriosis represent a large catchment area for CAM. In fact, as reported by Fisher et al patients affected were significantly more likely to consult a CAM practitioner or use CAM products compared to women without the disease. ${ }^{46}$

\section{Acupuncture and TENS}

In all studies, acupuncture had beneficial effects on pain intensity. In addition, patients reported an increase in social activities and school attendance in one study and an improvement in health-related QoL in the other two studies. None of the studies reported side effects. The overall results of the effects of acupuncture on pelvic pain related to endometriosis seem encouraging, particularly among adolescent women. However, no definitive conclusions can be drawn from these studies, due to the small sample sizes. ${ }^{44}$

TENS is a non-invasive and low-cost technique based on the use of mild electrical impulses applied to the intact surface of the skin with the objective of stimulating lowthreshold nerves to relieve pain and favor the release of endogenous opioids. TENS has been mentioned as alternative short-term pain management in women with dysmenorrhea in a consensus document on the management of endometriosis. ${ }^{44}$

\section{CONCLUSION}

Numerous cofactors can play a role in the progression and maintenance of endometriosis, which is thus a complex multifactorial disease.

Hormonal therapy represents the treatment of choice in patients with endometriosis; with this in mind, we could consider CAM a supplementary option to be added to hormonal treatment, or as a valuable opportunity for those women in whom medical therapy is contraindicated.

\section{Funding: No funding sources Conflict of interest: None declared Ethical approval: Not required}

\section{REFERENCES}

1. Kennedy S, Bergqvist A, Chapron C, D'Hooghe T, Dunselman G, Saridogan E, et al. ESHRE guideline on the diagnosis and management of endometriosis. Hum Reprod. 2005;20(10):2698-704.

2. Rock JA, Markham SM. Pathogenesis of endometriosis. Lancet. 1992;340:1264-7.

3. Giudice LC, Kao LC. Endometriosis. Lancet. 2004;364(9447):789-99.

4. Daly S (October 18, 2004). Endometrioma/Endometriosis. WebMD. Retrieved 2006.

5. Simoens S, Hummelshoj L, D'Hooghe T. Endometriosis: cost estimates and methodological perspective. Hum Reprod Update. 2007;13:394-404.

6. Huang HY. Medical treatment of endometriosis. Chang Gung Med J. 2008;31(5):431-40. 
7. Redwine DB. Conservative laparoscopic excision of endometriosis by sharp dissection: life table analysis and reoperation and persistent or recurrent disease. Fertil Steril. 1991;56:628-34.

8. Busacca M, Fedele L, Bianchi S, Candiani M, Agnoli B, Raffaelli R, Vignali M. Surgical treatment of recurrent endometriosis: laparotomy versus laparoscopy. Human reproduction (Oxford, England). 1998;13(8):2271-4.

9. Gambone JC, Mittman BS, Munro MG, Scialli, AR, Winkel CA. Chronic Pelvic Pain/Endometriosis Working Group. Consensus statement for the management of chronic pelvic pain and endometriosis: proceedings of an expert-panel consensus process. Fertil Steril. 2002;78:961-72.

10. The Practice Committee of the American Society for Reproductive Medicine American Society for Reproductive Medicine, Birmingham, Alabama Treatment of pelvic pain associated with endometriosis: a committee opinion. Fertil Steril. 2014;101(4):927-35.

11. Olive DL. Medical therapy of endometriosis. Semin Reprod Med. 2003;21:209-22.

12. Razzi S, Luisi S, Ferretti C, Calonaci F, Gabbanini M, Mazzini M. et al. Use of a progestogen-only preparation containing desogestrel in the treatment of recurrent pelvic pain after conservative surgery for endometriosis. Eur J Obstet Gynecol Reprod Biol. 2007; 135:188-90.

13. Vercellini P, Trespidi L, Colombo A, Vendola N, Marchini M, and Crosignani PG. A gonadotropinreleasing hormone agonist versus a low-dose oral contraceptive for pelvic pain associated with endometriosis. Fertil Steril. 1993;60:75-9.

14. Vercellini P, Frontino G, De Giorgi O, Pietropaolo G, Pasin R, and Crosignani PG. Continuous use of an oral contraceptive for endometriosis-associated recurrent dysmenorrhea that does not respond to a cyclic pill regimen. Fertil Steril. 2003;80:560-3.

15. Vercellini P, Cortesi I, and Crosignani PG. Progestogens for symptomatic endometriosis: a critical analysis of the evidence. Fertil Steril. 1997;68:393-401.

16. McCormack PL. Dienogest. A review of its use in the treatment of endometriosis. Drugs. 2010;70:2073-88.

17. Vercellini P, Frontino G, de Giorgi O, Aimi G, Zaina $\mathrm{B}$, and Crosignani PG. Comparison of a levonorgestrel-releasing intrauterine device versus expectant management after conservative surgery for symptomatic endometriosis: a pilot study. Fertil Steril. 2003;80:305-9.

18. Fedele L, Bianchi S, Zanconato G, Portuese A, and Raffaelli R. Use of a levonorgestrel-releasing intrauterine device in the treatment of rectovaginal endometriosis. Fertile Steril. 2001;75:485-8.

19. Lockhat FB, Emembolu JO, and Konje JC. The evaluation of the effectiveness of an intrauterineadministered progestogen (levonorgestrel) in the symptomatic treatment of endometriosis and in the staging of the disease. Hum Reprod. 2004;19:179-84.

20. BayogluTekin Y, Dilbaz B, Altinbas SK, and Dilbaz S. Postoperative medical treatment of chronic pelvic pain related to severe endometriosis: levonorgestrelreleasing intrauterine system versus gonadotropinreleasing hormone analogue. Fertil Steril. 2011;95: 492-6.

21. Brown J, Pan A, and Hart RJ. Gonadotrophinreleasing hormone analogues for pain associated with endometriosis. Cochrane Database Syst Rev. 2010;12:CD008475

22. Waller KG and Shaw RW. Gonadotropin-releasing hormone analogues for the treatment of endometriosis: long-term follow-up. Fertil Steril. 1993;59:511-5.

23. Barbieri RL. Endometriosis and the estrogen threshold theory: relation to surgical and medical treatment. J Reprod Med. 1998;43:287-92.

24. Pavone ME and Bulun SE. Aromatase inhibitors for the treatment of endometriosis. Fertil Steril. 2012;98:1370-9.

25. Farquhar C, Prentice A, Singla AA, Selak V. Danazol for pelvic pain associated with endometriosis. The Cochrane Library. 2007.

26. Ferrero S, Tramalloni D, Venturini PL, and Remorgida V. Vaginal danazol for women with rectovaginal endometriosis and pain sympotms persisting after insertion of a levonorgestrolreleasing intrauterine device. Int $\mathrm{J}$ Gynecol Obstet. 2011;113:116-9.

27. Mizutani T, Nishiyama S, Amakawa I, Watanabe A, Nakamuro K, and Terada N. Danazol concentrations in ovary, uterus, and serum and their effect on the hypothalamic-pituitary-ovarian axis during vaginal administration of a danazol suppository. Fertil Steril. 1995;63:1184-9.

28. Igarashi M, Iizuka M, Abe Y, and Ibuki Y. Novel vaginal danazol ring therapy for pelvic endometriosis, in particular deeply infiltrating endometriosis. Hum Reprod. 1998;13:1952-6.

29. Cobellis L, Razzi S, Fava A, Severi FM, Igarashi M, and Petraglia F. A danazol-loaded intrauterine device decreases dysmenorrhea, pelvic pain, and dyspareunia associated with endometriosis. Fertil Steril. 2004;82:239-40.

30. Davis L, Kennedy SS, Moore J, and Prentice A. Modern combined oral contraceptives for pain associated with endometriosis. Cochrane Database Syst Rev. 2007;3:CD001019.

31. Brown J, Kives S, and Akhtar M. Progestagens and anti-progestagens for pain associated with endometriosis. Cochrane Database Syst Rev. 2012;3:CD002122

32. Nawathe A, Patwardhan S, Yates D, Harrison GR, and Khan KS. Systematic review of the effects of aromatase inhibitors on pain associated with endometriosis. Br J Obstet Gynecol. 2008;115:81822. 
33. Soysal S, Soysal ME, Ozer S, Gul N, and Gezgin T. The effects of post-surgical administration of goserelin plus anastrozole compared to goserelin alone in patients with severe endometriosis: a prospective randomized trial. Hum Reprod. 2004;19:160-7.

34. Flower A, Liu JP, Lewith G, Little P, Li Q. Chinese herbal medicine for endometriosis. Cochrane Database Syst Rev. 2012;5:CD006568.

35. Lu D, Song H, Li Y, Clarke J, Shi G. Pentoxifylline for endometriosis. Cochrane Database Syst Rev (Online). 2012;1:CD007677.

36. Practice bulletin no. 114 management of endometriosis. Obstet Gynecol. 2010;116(1):223-36.

37. Laschke MW, Menger MD. Anti-angiogenic treatment strategies for the therapy of endometriosis. Human Reprod Update. 2012;18(6):682-702.

38. Canny GO, Lessey BA. The role of lipoxin A4 in endometrial biology and endometriosis. Mucosal Immunol. 2013;6(3):439-50.

39. Streuli I, de Ziegler D, Santulli P. An update on the pharmacological management of endometriosis. Expert Opin Pharmacother. 2013;14(3):291-305.

40. Valiani M, Ghasemi N, Bahadoran P, Heshmat R. The effects of massage therapy on dysmenorrhea caused by endometriosis. Iran J Nurs Midwifery Res. 2010;15(4):167-71.

41. Vercellini P, Buggio L, Berlanda N, Barbara G, Somigliana E, Bosari S. Estrogen-progestins and progestins for the management of endometriosis. Fertil Steril. 2016;106(7):1552-71.

42. Vercellini P, Viganò P, Somigliana E, Fedele L. Endometriosis: pathogenesis and treatment. Nat Rev Endocrinol. 2014;10(5):261-75.

43. Kong S, Zhang YH, Liu CF. The complementary and alternative medicine for endometriosis: a review of utilization and mechanism. Evid Based Complement Alternat Med. 2014;2014:146383.

44. Buggio L, Barbara G, Facchin F, Frattaruolo MP, Aimi G, and Berlanda N. Self-management and psychological-sexological interventions in patients with endometriosis: strategies, outcomes, and integration into clinical care. Int $\mathrm{J}$ Womens Health. 2017;9:281-93.

45. World Health Organization General guidelines for methodologies on research and evaluation of traditional medicine. 2000. Available from: http://apps.who.int/iris/bitstream/10665/66783/1/WH O_EDM_TRM_2000.1.pdf

46. Fisher C, Adams J, Hickman L, Sibbritt D. The use of complementary and alternative medicine by 7427 Australian women with cyclic perimenstrual pain and discomfort: a cross-sectional study. BMC Complement Altern Med. 2016;16:129.

Cite this article as: Ezzat L. Medical treatment of endometriosis: an update. Int J Reprod Contracept Obstet Gynecol 2017;6:4187-92. 